\title{
Linear Fractures Invisible on Routine Axial Computed Tomography: A Pitfall at Radiological Screening for Minor Head Injury
}

\author{
Kuniaki NAKAHARA, ${ }^{1}$ Satoru SHIMIZU, ${ }^{1}$ Takao KITAHARA, ${ }^{2}$ Hidehiro OKA, ${ }^{1}$ \\ Satoshi UTSUKI, ${ }^{1}$ Kazui SOMA, ${ }^{2}$ Shinichi KAN, ${ }^{3}$ and Kiyotaka FUJII ${ }^{1}$ \\ Departments of ${ }^{1}$ Neurosurgery, ${ }^{2}$ Critical Care and Emergency, and \\ ${ }^{3}$ Radiology, Kitasato University School of Medicine, Sagamihara, Kanagawa
}

\begin{abstract}
Computed tomography (CT) is now widely used as the only screening method for fractures in patients with head injury. However, clear depiction of a fracture requires a discontinuity in the skull, so linear fractures parallel to the CT slice may not be visualized. We retrospectively evaluated 302 patients with minor head injuries aged from 0 to 91 years, who had undergone routine skull radiography (anteroposterior and lateral views) and head CT to study these types of fracture and discuss the risk of nondetection. Three patients had linear fractures $(0.99 \%)$ that were invisible on bone window axial CT but detected on skull radiography, which all ran parallel to the scan slice. Two patients developed acute epidural hematoma or traumatic subarachnoid hemorrhage. Evaluation of head injury by only axial CT may miss such fractures and result in sequelae, so diagnosticians should be alert to the possible presence of this type of fracture.
\end{abstract}

Key words: computed tomography, head injury, linear fracture, misdiagnosis

\section{Introduction}

Minor head injury is characterized by a history of loss of consciousness, amnesia, or disorientation, and Glasgow Coma Scale (GCS) score of 13-15.1,2) The early diagnosis of cranial bone fractures by radiological examination is very important for predicting the possible occurrence of intracranial hematoma. Skull radiography is convenient and inexpensive, so remains important for radiological screening for minor head injury, ${ }^{1,2,4)}$ but the exclusive use of computed tomography (CT) for diagnostic purposes has increased. ${ }^{8)}$ We describe a type of linear fracture that is difficult to detect on bone window CT, to alert neurosurgeons to the diagnostic pitfalls.

\section{Materials and Methods}

The medical records were reviewed of 302 patients with minor head injury treated in the Department of Critical Care and Emergency Medicine or the

Received September 14, 2010; Accepted January 13, 2011
Department of Neurosurgery, Kitasato University Hospital, between January 2005 and August 2009. All patients underwent routine skull radiography (anteroposterior and lateral views, and Towne's view in cases with occipital impact) and head CT (brain and bone window in the axial plane) using a LightSpeed Ultra scanner (GE Healthcare, Waukesha, Wisconsin, USA), carried out within 24 hours of the injury, parallel to the orbitomeatal or anthropological basal line at 1-cm intervals in the supratentorial portion. Patients with penetrating skull injury or gunshot wounds were excluded. Patients with linear fractures that were invisible on bone window CT but detected on skull radiography were investigated.

\section{Results}

Skull fractures were identified in 39 of the 302 patients with minor head injury $(12.9 \%)$. Three of the 302 fractures $(0.99 \%)$ were invisible on bone window CT whereas skull radiography showed linear fractures running parallel to the CT slice plane without crossing the plane (Figs. 1-3). The patients with these fractures were aged from 3 months to 84 

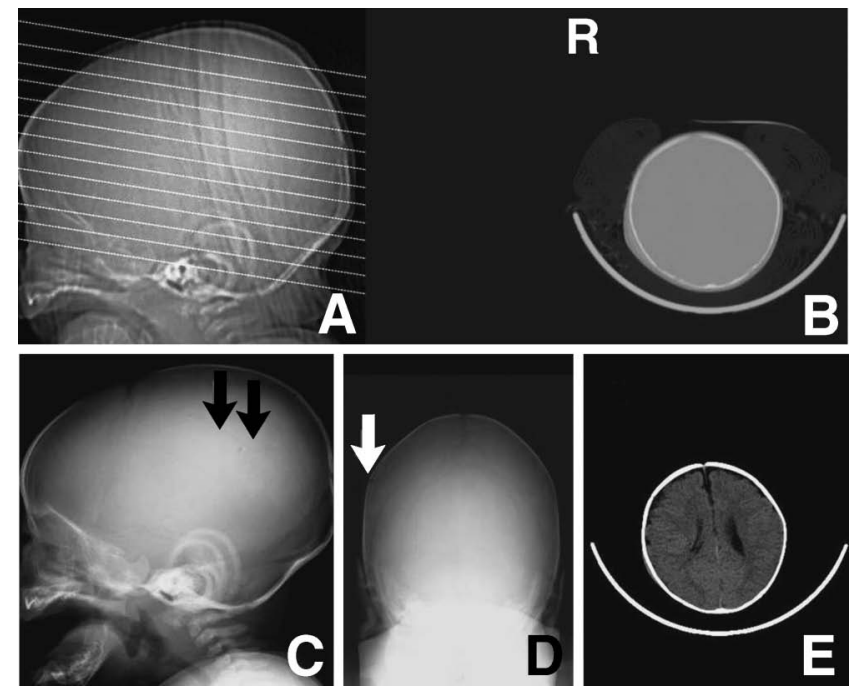

Fig. 1 Case 1 of a 3-month-old girl. Computed tomography (CT) scans, scout view (A) and axial bone window image (B), showing no obvious linear fracture. Skull radiographs, lateral view (C) showing a fracture line that did not cross the CT slice plane (arrows), and Towne's view (D) disclosing a fracture line (arrow). Axial brain window CT scan (E) showing no obvious hematoma.
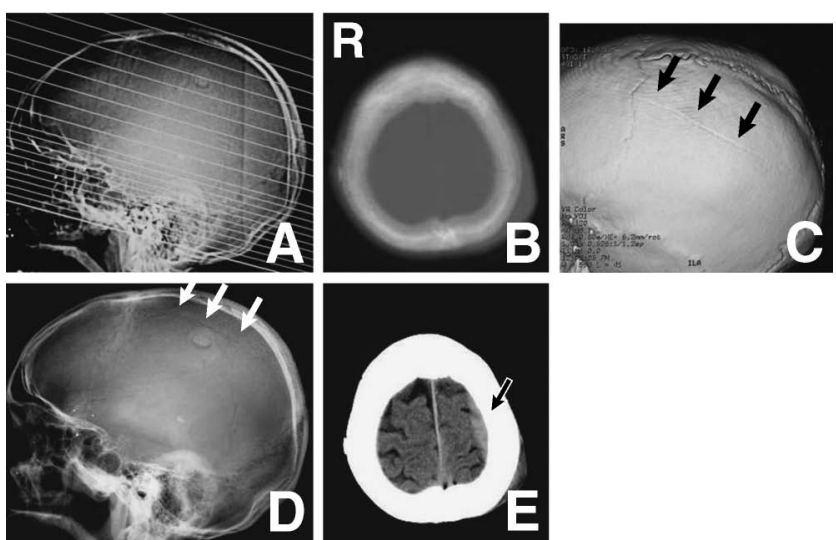

Fig. 2 Case 2 of a 72-year-old man with a history of surgically treated chronic subdural hematoma on the right. Computed tomography (CT) scans, scout view (A) and axial bone window image (B), showing no obvious linear fracture. Three-dimensional CT scan (C) clearly demonstrating a linear fracture in the parietal bone (arrows). Skull radiograph, lateral view (D), showing a fracture line that does not cross the CT slice plane (arrows). Axial brain window CT scan (E) disclosing acute epidural hematoma at the fracture site (arrow).

years; one was injured in a traffic accident and 2 fell or slipped. All three patients presented with subcutaneous hematoma in the region of impact. The
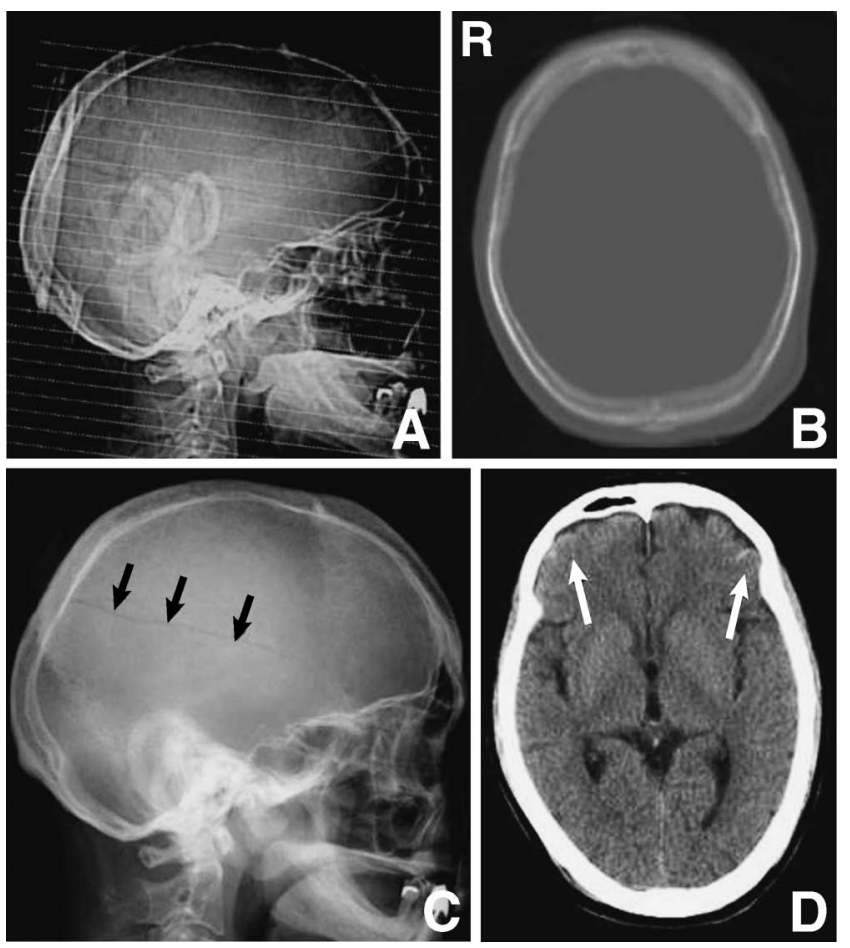

Fig. 3 Case 3 of an 84-year-old man. Computed tomography (CT) scans, scout view (A) and axial bone window image (B), showing no obvious linear fracture. Skull radiograph, lateral view (C), disclosing a fracture line that does not cross the CT slice plane (arrows). Axial brain window CT scan (D) showing traumatic subarachnoid hemorrhage (arrows).

GCS score at presentation was 15 in 2 patients and 12 in one. All fractures were located in the parietal bone. One patient had no intracranial lesions, one had acute epidural hematoma at the fracture site, and one had traumatic subarachnoid hemorrhage (Figs. 2E and 3D). All 3 patients were managed by observation and all had good outcomes.

\section{Discussion}

CT investigation of all patients with minor head injury, characterized by loss of consciousness, amnesia, disorientation, and GCS of $13-15$, is a significant economic burden.5) Therefore, attempts have been made to identify clinical factors indicative of intracranial lesions, and guidelines for the evaluation of minor head injury have been published for both general and pediatric populations.7) The rate of fracture in patients with minor head injury and GCS of 13 was much higher than in patients with GCS of 15 (18\% vs. $3.8 \%$ ), suggesting that the acquisition of CT scans is important in patients with lower GCS. ${ }^{3)}$ In Japan, most patients with minor head injury un- 
dergo CT as the first imaging study, primarily because of the requests of patients and their families, and also because of institutional protocols to protect against law suits. Skull fractures are easily detected on bone window CT, so many patients with minor head injury are subjected only to CT.

The information about skull base fractures provided by CT is superior to that from radiography. However, linear fractures running parallel to the CT slice without crossing the slice plane may not be detected. Three-dimensional CT scans were of diagnostic value in one of our 3 cases. Based on our experience we strongly suggest that despite the value of CT, radiography should not be abandoned in patients with head trauma. This conventional examination can be performed in almost all hospitals and the acquisition time is short. If the fracture side of the skull convexity is unclear, we recommend the acquisition of both right and left lateral skull radiographs and comparison of the sharpness of the fracture line. The image with the sharper line identifies the side of the fracture. The delivered radiation dose is higher for skull radiography than CT ( 50 mGy vs. $2.01 \mathrm{mGyj}{ }^{6)}$ Nonetheless, we recommend the additional acquisition of skull radiographs in patients with suspected skull fractures that may be occult on CT.

This study demonstrates the presence of linear fractures on radiography that were invisible on axial CT, and suggests that patients with head injury should not be investigated only by CT. The incidence of these types of fracture is low, but if overlooked, patients with even minor head trauma may suffer serious consequences.

\section{References}

1) Baker HL Jr: The impact of computed tomography on neuroradiologic practice. Radiology 116: 637-640, 1975

2) Baker HL Jr: Computed tomography and neuroradiology: A fortunate primary union. AJR Am J Roentgenol 127: 101-110, 1976

3) Culotta VP, Sementilli ME, Gerold K, Watts CC: Clinicopathological heterogeneity in the classification of mild head injury. Neurosurgery 38: 245-250, 1996

4) Larson EB, Omenn GS, Margolis MT, Loop JW: Impact of computed tomography on utilization of cerebral angiograms. AJR Am J Roentgenol 129: 1-3, 1977

5) Stiell IG, Wells GA, Vandemheem K, Clement C, Lesiuk H, Laupacis A, McKnight RD, Verbeek R, Brison R, Cass D, Eisenhauer ME, Greenberg G, Worthington J: The Canadian CT Head Rule for patients with minor head injury. Lancet 357: 1391-1396, 2001

6) Suzuki S: [The interim report No. 2 of investigative research on patient exposure doses in diagnostic X-ray examinations]. Nippon Hoshasen Gijutsu Gakkai Zasshi 65: 1582-1589, 2009 (Japanese)

7) Tress BM: The need for skull radiography in patients presenting for CT. Radiology 146: 87-89, 1983

8) Weinstein MA, Alfidi RJ, Duchesneau PM: Computed tomography versus skull radiography. AJR Am J Roentgenol 128: 873, 1977

Address reprint requests to: Kuniaki Nakahara, MD, Department of Neurosurgery, Kitasato University School of Medicine, 1-15-1 Kitasato, Minami-ku, Sagamihara, Kanagawa 252-0375, Japan. e-mail: nakahara19@hotmail.com 